Pesquisa Florestal Brasileira
Brazilian Journal of Forestry Research
https://www.embrapa.br/pfb

\title{
Efeito de homeopatia na germinação de sementes de ipê amarelo
}

\author{
Naira Maranhão Silva, Bianca de Oliveira1 ${ }^{1}$, Stefany Lorrayny Lima1 \\ ${ }^{1}$ Universidade do Estado de Mato Grosso, BR 158, Km 148, CEP 78690-000, Nova Xavantina, MT, Brasil
}

\author{
"Autor correspondente: \\ controladoria.pira@grupoitaquere.com.br \\ Termos para indexação: \\ Tabebuia serratifolia \\ Calcarea carbonica \\ Carbo vegetabilis \\ Silicea \\ Handroanthus serratifolius \\ Index terms: \\ Tabebuia serratifolia \\ Calcarea carbonica \\ Carbo vegetabilis \\ Silicea \\ Handroanthus serratifolius
}

Histórico do artigo:

Recebido em 28/05/2013

Aprovado em 23/09/2014

Publicado em 10/10/2014

doi: 10.4336/2014.pfb.34.79.540

\begin{abstract}
Resumo - Estudos com homeopatia na germinação de sementes de espécies florestais nativas são escassos, apesar do potencial dessa tecnologia de baixo impacto. Por esse motivo, o objetivo desse trabalho foi avaliar o uso de medicamentos homeopáticos em diferentes dinamizações na germinação de sementes de ipê amarelo. O delineamento experimental foi inteiramente casualizado com três repetições, em esquema fatorial, com três medicamentos em cinco dinamizações, totalizando 16 tratamentos com a testemunha formada por água destilada. Foram utilizados os medicamentos homeopáticos Calcarea carbonica, Carbo vegetabilis e Silicea, todos nas dinamizações 6, 12, 30, 100 e 200 centesimais hahnemanianas $(\mathrm{CH})$. Em relação à porcentagem de germinação e ao índice de velocidade de germinação a homeopatia de Silicea na dinamização $12 \mathrm{CH}$ foi menos eficiente quando comparada às demais dinamizações e aos demais medicamentos. $\mathrm{O}$ uso dos preparados homeopáticos não beneficia o padrão de germinação de sementes de ipê amarelo.
\end{abstract}

\section{Evaluation of homeopathy in seed germination of yellow ipe}

\begin{abstract}
Studies with homeopathie on seed germination of native forest species are scarce, regardless of its potential as low impact technology. Therefore, the objective of this study was to evaluate the use of homeopathic medications in different dynamizations in the seed germination of yellow ipe. The experimental design was completely randomized with three replications, in a factorial scheme with the three medicines in five dynamizations, totaling 16 treatments with the control consisting of distilled water. The medications used were Calcarea carbonica, Carbo vegetabilis and Silicea. The mediations were applied in of 6, 12, 30, 100 and 200 centesimals hahnemanianas $(\mathrm{CH})$ dynamizations. Regarding germination percentage and speed of germination index the medicament Silicea in $12 \mathrm{CH}$ dynamization was less efficient when compared to others medications and dynamizations. The use of homeopathic preparations does not benefit the pattern of yellow ipe seeds germination.
\end{abstract}

\section{Introdução}

Devido à importância da preservação de espécies nativas, bem como a necessidade de suprir a demanda por reflorestamento de áreas degradadas, com formas de produção sustentáveis, o uso de técnicas eficientes e com menor impacto ambiental tem se tornado essencial. Nesse contexto, o uso de homeopatia em plantas tem se mostrado uma alternativa para sistemas de produção mais eficientes, sendo oficializada como insumo agrícola pelo Ministério da Agricultura e do Abastecimento, na Instrução Normativa $n^{\circ}$ 64, de 18 de dezembro de 2008 (Brasil, 2008).

Os estudos em homeopatia se fundamentam em quatro princípios: semelhança, doses diluídas e dinamizadas, utilização de um único preparado por veze experimentação em indivíduos sadios. Essas substâncias curam sintomas que são capazes de produzir em indivíduos sadios, quando experimentadas isoladamente 
e em doses diluídas e dinamizadas (Andrade et al., 2001). Medicamentos homeopáticos em plantas promovem maior resistência a ambientes instáveis ou extremos, estimulam o sistema de defesa dos vegetais em fase germinativa e a quebra de dormência de sementes (Arruda et al., 2005; Carvalho et al., 2005).

Os medicamentos homeopáticos são selecionados de acordo com suas indicações para estresses em plantas, como o Carbo vegetabilis que auxilia no reequilíbrio das plantas submetidas a geadas, na quebra de dormência de algumas sementes, na absorção dos nutrientes, aumenta a resistência ao estresse hídrico e mudanças de temperatura, além de ser utilizado para o tratamento de doenças fúngicas (Tichavsky, 2007). Conforme o mesmo autor, o medicamento Calcarea carbonica é indicado para plantas sensíveis que se encontram em condições adversas, além de problemas como crescimento lento, auxiliando na regulação da germinação e crescimento das plantas. Enquanto que o medicamento Silicea é indicado para estimular o crescimento vegetativo, contra ataques de patógenos, baixa absorção de nutrientes e para plantas que apresentam danos na epiderme.

No cultivo de espécies nativas, as sementes são o modo de propagação mais empregado para a produção de mudas e a busca de conhecimentos sobre as condições que auxiliem o processo de germinação são importantes para garantir plantas em quantidade e qualidade para implantação em campo, garantindo o sucesso do plantio e, consequentemente, conservação das espécies. Entre as espécies nativas, o ipê amarelo (Handroanthus serratifolius (A. H. Gentry) S. Grose) é uma espécie arbórea pertencente à família Bignoniaceae, encontrada em quase todo território brasileiro. É característica de florestas tropicais úmidas, região amazônica, atlântica, florestas estacionais, cerrado sentido restrito e cerradões. Pode ser utilizada em reflorestamentos com finalidades comerciais e em projetos de recuperação de áreas degradadas, de matas ciliares e demais áreas de preservação permanente (Lorenzi, 2008).

A germinação da espécie é fácil, apresentando altas taxas germinativas, mas o período de viabilidade das sementes é curto, dificultando o estabelecimento de técnicas de cultivo para silvicultura e reflorestamentos de áreas degradadas, além de limitar sua longevidade natural (Silva Junior, 2005; Lorenzi, 2008).

Alguns trabalhos comprovam a eficiência do uso de medicamentos homeopáticos sobre o crescimento e produtividade vegetal (Grisa et al., 2007; Baumgartner et al., 2008; Ferreira et al., 2009) e no controle de pragas e doenças (Betti et al., 2003; Rossi et al., 2007; Carneiro et al., 2011), mas apenas alguns trabalhos observam o efeito desses medicamentos em sementes (Binder et al., 2005; Marques et al., 2008; Bonfim et al., 2010), ou abordam apenas espécie de cultivo anuais, sendo escassos estudos com espécies florestais. Com isso, este trabalho tem como objetivo avaliar o efeito de diferentes preparados homeopáticos na germinação de sementes de ipê amarelo para verificar se estes afetam sementes sadias.

\section{Material e métodos}

A pesquisa foi conduzida no Laboratório de Química da Universidade do Estado de Mato Grosso (UNEMAT),

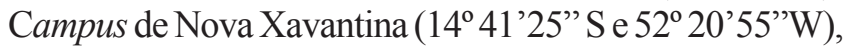
em 2011. As sementes de ipê amarelo foram retiradas da coleção de sementes de espécies nativas do herbário da UNEMAT, coletadas em 2011 em diferentes plantas matrizes do Parque Municipal do Bacaba, que apresenta uma vegetação de transição Cerrado-Floresta Amazônica (Marimon et al., 2006), e armazenadas em ambiente refrigerado para conservação de seu potencial germinativo.

$\mathrm{O}$ delineamento experimental foi inteiramente casualizado com três repetições, em esquema fatorial, com três medicamentos em cinco dinamizações, totalizando 16 tratamentos com o controle formado por água destilada. As soluções homeopáticas foram obtidas com água destilada, $0,5 \%$ de álcool e o princípio ativo de cada medicamento, Calcarea carbonica $(\mathrm{Cc})$, Carbo vegetabilis $(\mathrm{Cv})$ ou Silicea $(\mathrm{Si})$, obtidos em farmácia homeopática artesanal. Cada medicamento foi preparado nas dinamizações $6,12,30,100$ e 200 centesimais hahnemanianas $(\mathrm{CH})$. Para cada tratamento utilizou-se 18 sementes, colocadas em béqueres de $200 \mathrm{~mL}$, previamente esterilizados em autoclave a $150{ }^{\circ} \mathrm{C}$.

Em cada béquer foram adicionados $100 \mathrm{~mL}$ de solução (85 $\mathrm{mL}$ de água destilada $+15 \mathrm{~mL}$ de homeopatia), deixando a semente em embebição por 24 horas. Após este período, as sementes foram distribuídas em papel de germinação ("germitest") umedecido com água (2,5 vezes o peso do substrato seco) e colocadas em estufa Quimis com temperatura entre $30^{\circ} \mathrm{C}$ e $32{ }^{\circ} \mathrm{C}$, conforme descrito por Ferreira et al. (2002) e Stockman et al. (2007) para teste de germinação de Tabebuia sp. As sementes foram avaliadas diariamente, até a estabilização da germinação, adotando-se os critérios para a análise de sementes indicados em Brasil (2009). 
Calculou-se a porcentagem de germinação e o índice de velocidade de germinação, conforme Maguire (1962). Para analisar se houve diferença entre os tratamentos foram realizadas análises de variância (ANOVA), sendo, posteriormente, verificada a significância das variações pelo teste de Duncan a 5\% de probabilidade.

\section{Resultados e discussão}

Para os preparados homeopáticos de Silicea a germinação das sementes de ipê foi menos eficiente na dinamização $12 \mathrm{CH}$ quando comparada às demais, onde houve uma redução de $44 \%$, aproximadamente (Figura 1). As dinamizações dos medicamentos Calcarea carbonica $(\mathrm{p}=0,58)$ e Carbo vegetabilis $(\mathrm{p}=0,72)$ não mostraram diferenças entre si, evidenciando que nas condições testadas esses medicamentos não influenciam na germinação de sementes de ipê amarelo.

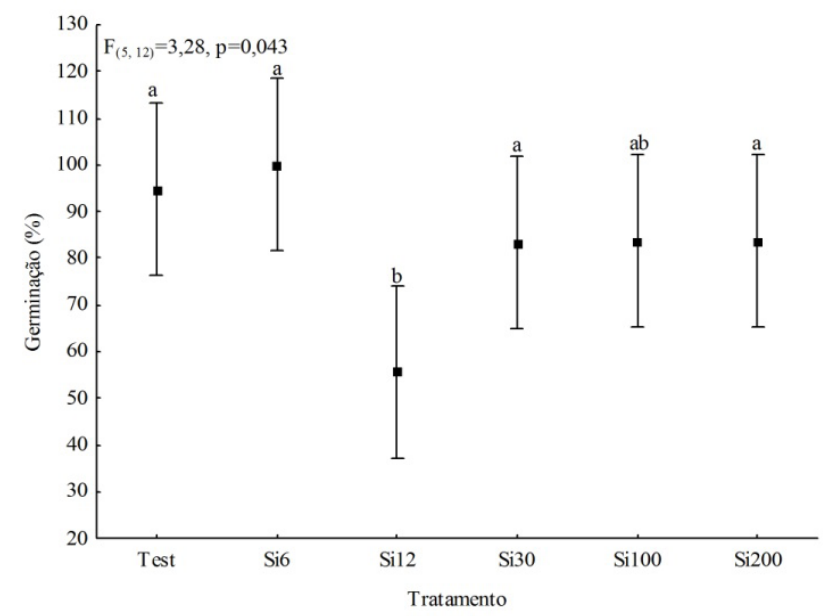

Figura 1. Germinação total (\%) de sementes de ipê amarelo (H. serratifolius) submetidas a cinco dinamizações do medicamento homeopático Silicea $(\mathrm{Si})$. Médias seguidas pela mesma letra não diferem entre si pelo teste de Duncan a 5\% de probabilidade.

Comparando os medicamentos homeopáticos, observase que não houve diferenças para a germinação das sementes de ipê para as dinamizações $6 \mathrm{CH}(\mathrm{p}=0,34)$, $30 \mathrm{CH}(\mathrm{p}=0,68), 100 \mathrm{CH}(\mathrm{p}=0,36)$ e $200 \mathrm{CH}(\mathrm{p}=0,64)$, enquanto que na dinamização $12 \mathrm{CH}$ o medicamento Silicea apresentou, em média, redução de $38 \%$ na germinação, quando comparado aos demais (Figura 2).

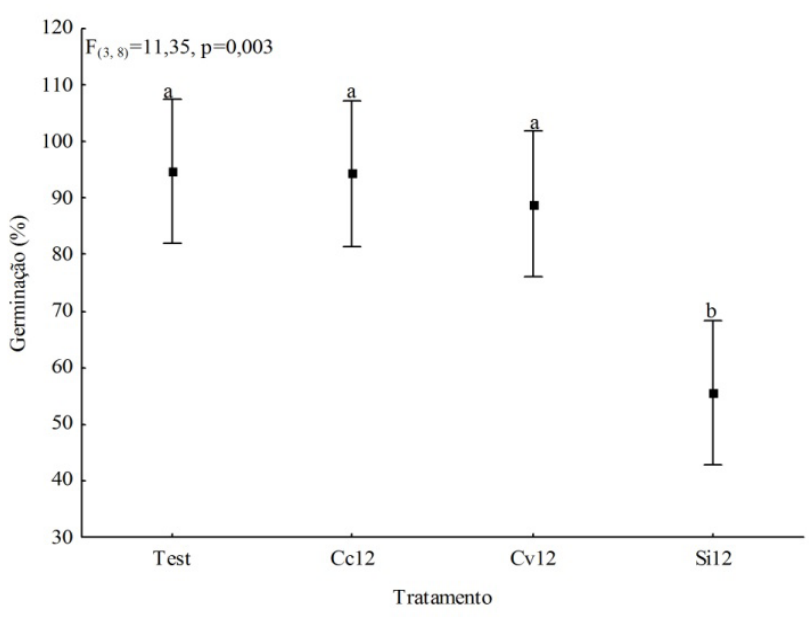

Figura 2. Germinação total (\%) de sementes de ipê amarelo (H. serratifolius) submetidas a preparados homeopáticos dos medicamentos Calcaria carbonica (Cc), Carbo vegetabilis (Cv) e Silicea (Si), na dinamização $12 \mathrm{CH}$. Médias seguidas pela mesma letra não diferem entre si pelo teste de Duncan a $5 \%$ de probabilidade.

O índice de velocidade de germinação também foi semelhante nas dinamizações dos medicamentos homeopáticos Calcarea carbonica $(\mathrm{p}=0,097)$ e Carbo vegetabilis $(\mathrm{p}=0,21)$, corroborando com os resultados observados para a porcentagem de germinação. Foram observadas diferenças entre as dinamizações para o medicamento Silicea, onde todas mostraram resultados inferiores ao tratamento controle, que apresentou em média, um acréscimo de $9 \%$ no IVG (Figura 3). Também se observa que a dinamização $12 \mathrm{CH}$ apresentou o menor desempenho, com uma redução de $10 \%$, em média, quando comparada às demais.

Para os resultados de índice de velocidade de germinação entre os medicamentos homeopáticos, observa-se que não houve diferenças para as dinamizações $6 \mathrm{CH}(\mathrm{p}=0,057)$ e $30 \mathrm{CH}(\mathrm{p}=0,21)$. No entanto, o IVG de $12 \mathrm{CH}$ foi superior para o tratamento controle, que apresenta um aumento médio de $10 \%$, quando comparado aos medicamentos que mostraram melhores resultados (Cc e $\mathrm{Cv}$ ) (Figura 4). Nessa dinamização também se observa que o medicamento Silicea mostrou uma diminuição no IVG de $9 \%$, em média, quando comparado aos demais. Para a dinamização $100 \mathrm{CH}$ o tratamento controle mostrou maior valor de IVG, com um aumento médio de $10 \%$ quando comparado aos demais, mas foi semelhante 
ao medicamento Cc (Figura 5). O IVG do tratamento controle na dinamização $200 \mathrm{CH}$ apresentou melhor resultado, com um acréscimo médio de $10 \%$ em relação aos outros medicamentos testados (Figura 6).

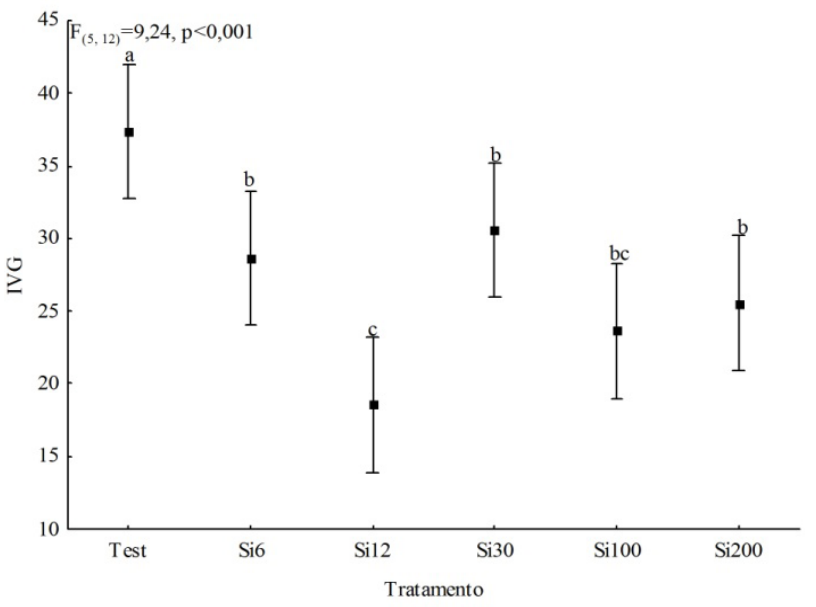

Figura 3. Índice de velocidade de germinação (IVG) de sementes de ipê amarelo (H. serratifolius) submetidas a cinco dinamizações do medicamento homeopático Silicea (Si). Médias seguidas pela mesma letra não diferem entre si pelo teste de Duncan a $5 \%$ de probabilidade.

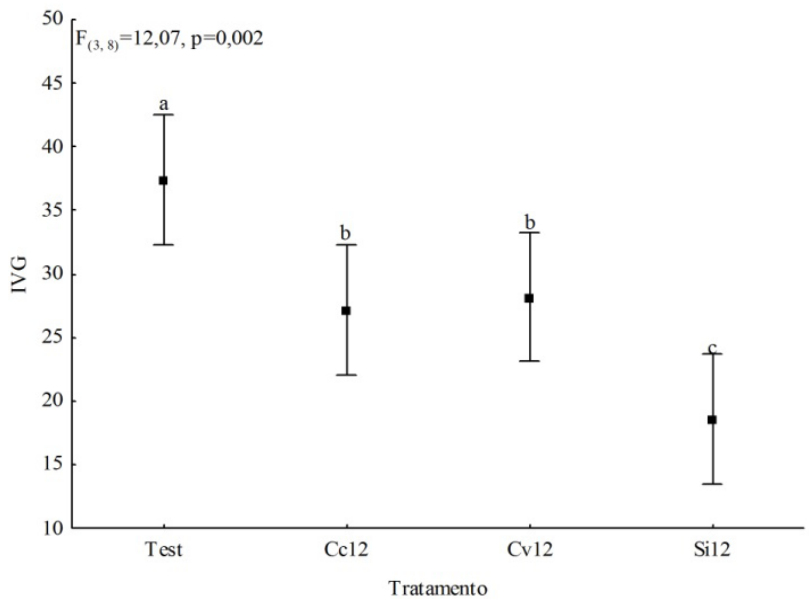

Figura 4. Índice de velocidade de germinação (IVG) de sementes de ipê amarelo (H. serratifolius) submetidas a três preparados homeopáticos dos medicamentos Calcaria carbonica $(\mathrm{Cc})$, Carbo vegetabilis $(\mathrm{Cv})$ e Silicea $(\mathrm{Si})$ na dinamização $30 \mathrm{CH}$. Médias seguidas pela mesma letra não diferem entre si pelo teste de Duncan a $5 \%$ de probabilidade.

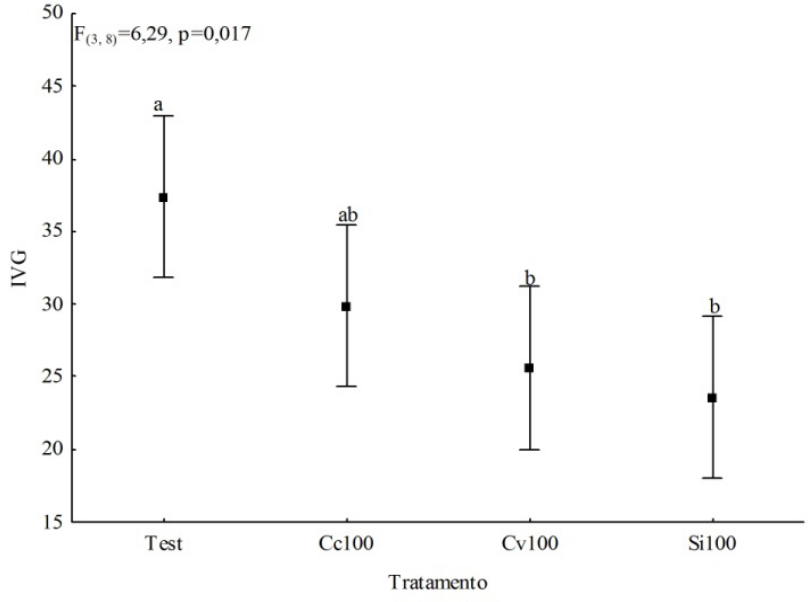

Figura 5. Índice de velocidade de germinação (IVG) de sementes de ipê amarelo (H. serratifolius) submetidas a três preparados homeopáticos dos medicamentos Calcaria carbonica $(\mathrm{Cc})$, Carbo vegetabilis $(\mathrm{Cv})$ e Silicea $(\mathrm{Si})$ na dinamização $100 \mathrm{CH}$. Médias seguidas pela mesma letra não diferem entre si pelo teste de Duncan a 5\% de probabilidade.

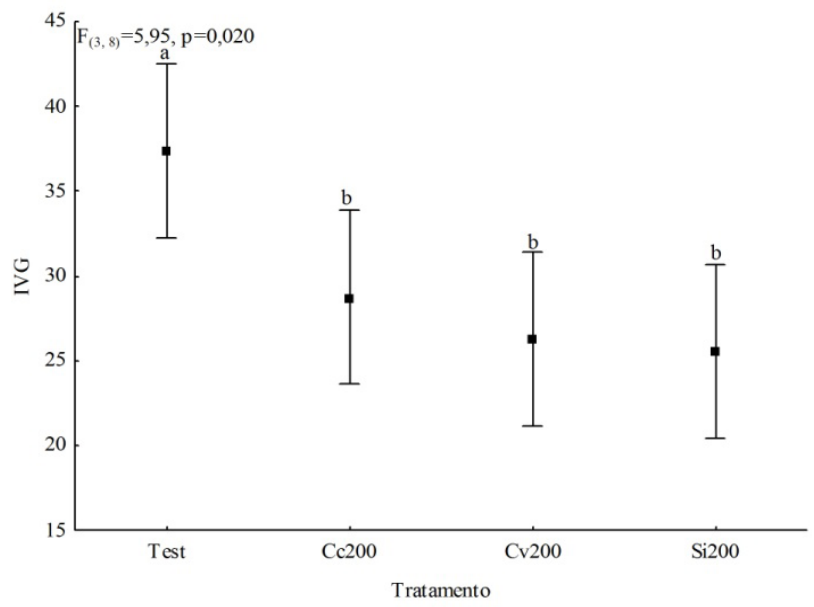

Figura 6. Índice de velocidade de germinação (IVG) de sementes de ipê amarelo (H. serratifolius) submetidas a três preparados homeopáticos dos medicamentos Calcaria carbonica $(\mathrm{Cc})$, Carbo vegetabilis $(\mathrm{Cv})$ e Silicea $(\mathrm{Si})$ na dinamização $200 \mathrm{CH}$. Médias seguidas pela mesma letra não diferem entre si pelo teste de Duncan a 5\% de probabilidade.

Os resultados apontam que os preparados homeopáticos não afetam positivamente o padrão de germinação das sementes de ipê amarelo, mas evidenciam que a dinamização $12 \mathrm{CH}$ do medicamento Silicea age como inibidora. Andrade et al. (2012) discutem que o uso da 
homeopatia pode causar alterações no padrão fisiológico vegetal, fazendo com que apresente efeitos potenciais em resposta às alterações causadas pela aplicação do medicamento, sendo que essas respostas podem ser tanto positivas como negativas.

Brizzi et al. (2000), avaliando homeopatia de arsênico em sementes de trigo, e Marques et al. (2008), em trabalho com homeopatia de citronela em Sida rhombifolia, também observaram efeitos distintos em relação às diferentes dinamizações, podendo induzir ou inibir a germinação e influenciar o índice de velocidade de germinação. Efeitos positivos em sementes de alface expostas a níveis tóxicos de alumínio foram observados por Bonfim et al. (2010), com o medicamento Calcarea carbonica nas dinamizações $12 \mathrm{CH}$ e $6 \mathrm{CH}$, mas também verificaram diferenças em relação as dinamizações.

Devido à grande biodiversidade vegetal e diferentes processos fisiológicos das espécies, bem como diferentes preparos homeopáticos e dinamizações que podem ser utilizados, são necessários estudos com diferentes medicamentos, dinamizações e espécies vegetais nas diferentes fases de desenvolvimento para orientar o uso dessa prática nos sistemas de produção vegetal. Em geral os testes com medicamentos homeopáticos são realizados em sementes que apresentem algum fator de estresse (Brizzi et al., 2000; Hamman et al., 2003; Bonfim et al., 2010). Assim, os resultados de ineficiência do uso de tratamentos homeopáticos na germinação das sementes de ipê amarelo podem estar relacionados às boas condições da semente e ao elevado potencial germinativo dessa espécie (Lorenzi, 2008; Oliveira, 2012), não havendo necessidade de estímulos externos para promover uma melhoria no padrão de germinação.

As características de variabilidade genética da espécie vegetal também podem influenciar os resultados observados quando as sementes são submetidas aos medicamentos homeopáticos. Sobre isso Betti et al. (1994) discutem, em trabalho sobre homeopatia de Arsenicum album em sementes de trigo, que os resultados não são derivados apenas das respostas genéticas das sementes, mas do efeito das doses da homeopatia nas características de germinação, sustentando a ideia de que os tratamentos homeopáticos promovem alterações fisiológicas nas plantas. Além da variabilidade genética, fatores externos como a temperatura podem influenciar nos resultados encontrados. No entanto, trabalhos com o gênero Tabebuia concluem que temperaturas entre $25^{\circ} \mathrm{C}$ e $35^{\circ} \mathrm{C}$ são as mais adequadas para a germinação das sementes (Santos et al., 2005; Oliveira et al., 2012), indicando que os resultados não sofreram interferências dos fatores externos.

Apesar de já existirem medicamentos com indicação comprovada para aplicação em plantas sob estresse (Tichavsky, 2007), novos estudos com uso de homeopatia em plantas ainda são necessários, devido às diferentes respostas das espécies aos fatores de estresse, além das substâncias e dinamizações usadas como homeopatia e a forma e tempo de avaliação, que são fatores que devem ser ajustados para se obter respostas consistentes da aplicação dessa técnica na agricultura (Jäger et al., 2011).

\section{Conclusões}

O uso dos preparados homeopáticos dos medicamentos Calcarea carbonica, Carbo vegetabilis e Silicea não beneficia o padrão de germinação de sementes de ipê amarelo.

A homeopatia de Silicea na dinamização $12 \mathrm{CH}$ age como inibidor da germinação de sementes de ipê amarelo.

\section{Referências}

ANDRADE, F. M. C.; CASALI, V. W. D.; CECON, P. R. C. Crescimento e produção de cumarina em plantas de chambá (Justicia pectoralis Jacq.) tratadas com isoterápico. Revista Brasileira Plantas Medicinais, Botucatu, v. 14, p. 154-158, 2012.

ANDRADE, F. M.; CASALI, V. W. D.; De VITA, B.; CECON, P. R.; BARBOSA, L. C. A. Efeito de homeopatias no crescimento e na produção de cumarina em chambá (Justicia pectoralis Jacq.). Revista Brasileira de Plantas Medicinais, Botucatu, v. 4, n. 1, p. 19-28, 2001.

ARRUDA, V. M.; CUPERTINO, M. C.; LISBOA, S. P.; CASALI, V. W. D. Homeopatia triuma na agronomia: as propostas de Roberto Costa e algumas relações com o agroecossistema. Viçosa, MG: Ed. UFV, 2005. 119 p.

BAUMGARTNER, S.; SCHALLER, S. D.; KAMPFER, J.; THURNEYSEN, A. U.; HEUSSER, P. Reproducibility of dwarf pea shoot growth stimulation by homeopathic potencies of gibberellic acid. Complementary Therapies in Medicine, Edinburgh, v. 16, p. 183-191, 2008. DOI: http://dx.doi.org/10.1016/j.ctim.2008.03.001

BETTI, L.; BRIZZI, M.; NANI, M. D.; PERUZZI, M. A pilot statistical study with homoeopathic potencies of Arsenicum album in wheat germination as a simple model. The British Homoeopathic Journal, São Paulo, SP, v. 83, n. 4, p. 195-201, 1994.

BETTI, L.; LAZZARATO, L.; TREBBI, G.; BRIZZI, M.; CALZONI, G. L.; BORGHINI, F.; NANI, D. Effects of homeopathic arsenic on tobacco plant resistence to tobacco mosaic virus. Theoretical suggestions about system variability, base on a large experimental data set. Homeopathy, São Paulo, SP, v. 92, p. 195-202, 2003. DOI: http://dx.doi.org/10.1016/j.homp.2003.08.014 
BINDER, M.; BAUMGARTNER, S.; THURNEYSEN, A. The effects of a 45x Potency of Arsenicum album on wheat seedling growth: a Reproduction trial. Forsch Komplementärmed Klass Naturheilkd, Germania, v. 12, p. 284-291, 2005. DOI: http://dx.doi. org/10.1159/000087969

BONFIM, F. P. G.; DORES, R. G. R.; MARTINS, E. R.; CASALI, V. W. D. Germination and vigor of lettuce seeds (Lactuca sativa L.) pelleted with homeopathic preparations Alumina and Calcarea carbonica subjected to toxic levels of aluminum. International Journal of High Dilution Research, Brasília, DF, v. 9, n. 33, p. 138-146, 2010.

BRASIL. Ministério da Agricultura, Pecuária e Abastecimento. Instrução Normativa ${ }^{\circ}$ 64, de 18 de dez. 2008. Aprova o Regulamento Técnico para os Sistemas Orgânicos de Produção Animal e Vegetal. Diário Oficial [da] República Federativa do Brasil, Brasília, DF, 19 de dez. de 2008, Seção 1, p. 21.

BRASIL. Ministério da Agricultura, Pecuária e Abastecimento. Secretaria de Defesa Agropecuária. Regras para análise de sementes. Brasília, DF, 2009. 395 p.

BRIZZI, M.; NANI, D.; PERUZZI, M.; BETTI, L. Statistical analysis of the effect of high dilutions of arsenic in a large dataset from a wheat germination model. The British Homeopathic Journal, São Paulo, SP, v. 89, n. 2, p. 63-67, 2000.

CARNEIRO, S. M. T. P. G.; ROMANO, E. D. B.; GARBIM, T. H. S.; OLIVEIRA, B. G.; TEIXEIRA, M. Z. Pathogenetic trial of boric acid in bean and tomato plants. International Journal of High Dilution Research, Brasília, DF, v. 10, n. 34, p. 37-45, 2011.

CARVALHO, L. M.; CASALI, V. W. D.; LISBOA, S. P.; SOUZA, M. A.; CECON, P. R. Efeito da homeopatia Arnica montana, nas potências centesimais, sobre plantas de artemísia. Revista Brasileira de Planta Medicinal, Botucatu, v. 7, n. 3, p. 33-36, 2005.

FERREIRA, C. M.; ALMIR, J. O.; DAVIDE, A. C.; MENDES, R. G. Metodologia para a condução do teste de germinação em sementes de ipê-amarelo (Tabebuia serratifolia (Vahl) Nicholson). Cerne, Lavras, v. 8, n. 2, p. 17-25, 2002.

FERREIRA, I. C. P. V.; ARAÚJO, A. V.; GOMES, J. G.; SALES, N. L. P. Preparados homeopáticos, extrato de bartimão e urina de vaca: alternativas para o controle da fusariose do abacaxi. Revista Brasileira de Agroecologia, Porto Alegre, v. 4, n. 2, p. 2948-2951, 2009.

GRISA, S.; TOLEDO, M. V.; OLIVEIRA, L. C.; HOLZ, L.; MARINE, D. Crescimento e produtividade de alface sob diferentes diluições do medicamento homeopático Arnica montana. Revista Brasileira de Agroecologia, Porto Alegre, v. 2, n. 2, p. 1050-1053, 2007.

HAMMAN, B.; KONING, G.; HIM LOK, K. Homeopathically prepared gibberellic acid and barley seed germination. Homeopathy, São Paulo, SP, v. 92, p. 140-144, 2003. DOI: http://dx.doi. org/10.1016/S1475-4916(03)00045-6
JÄGER, T.; SCHERR, C.; SHAH, D.; MAJEWSKY, V.; BETTI, L.; TREBBI, G.; BONAMIN, L.; SIMÕES-WÜST, A. P.; WOLF, U.; SIMON, M.; HEUSSER, P.; BAUMGARTNER, S. Use of homeopathic preparations in experimental studies with abiotically stressed plants. Homeopathy, São Paulo, SP, v. 100, p. 275-287, 2011. DOI: http://dx.doi.org/10.1016/j.homp.2011.05.008

LORENZI, H. Árvores brasileiras: manual de identificação e cultivo de plantas arbóreas nativas do Brasil. Rio de Janeiro, RJ: Nova Odessa: Plantarum, 2008. 384 p.

MAGUIRE, J. D. Speeds of germination-aid selection and evaluation for seedling emergence and vigor. Crop Science, Madison, v. 2, p. 176-7, 1962.

MARIMON, B. S.; LIMA, E. S.; DUARTE, T. G.; CHIEREGATTO, L. C.; RATTER, J. A. Observations on the vegetation of northeastern Mato Grosso, Brazil, IV an analysis of the Cerrado-Amazonian forest ecotone. Edinburgh Journal of Botany, Edinburgh, v. 63, p. 323341, 2006. DOI: http://dx.doi.org/10.1017/S0960428606000576

MARQUES, R. M.; MARQUES-SILVA, G. G.; BONATO, C. M. Effect of high dilutions of Cimbopogon winteranus Jowitt (citronella) on the germination and growth of seedlings of Sida rhombifolia. International Journal of High Dilution Research, Brasília, DF, v. 7, n. 22, p. 31-35, 2008.

OLIVEIRA, O. S. Tecnologia de sementes florestais: espécies nativas. Curitiba: Ed. da UFPR, 2012. 389 p.

ROSSI, F.; MELO, P. C. T.; PASCHOLATU, S. F.; CASALI, V. W. D.; AMBROSANO, E. J.; GUIRADO, N.; MENDES, P. C. D.; AMBROSANO, G. M. B.; SCHAMMASS, E. A.; TOFFANO, L.; DI PIERO R. M. Aplicação de bioterápico visando induzir resistência em tomateiro contra mancha bacteriana. Revista Brasileira de Agroecologia, Porto Alegre, v. 2, n. 1, p. 858-861, 2007.

SANTOS, D. L.; SUGAHARA, V. Y.; TAKAKI, M. Efeitos da luz e da temperatura na germinação de sementes de Tabebuia serratifolia (Vahl) Nich, Tabebuia chrysotricha (Mart. ex DC.) Standl. e Tabebuia roseoalba (Ridl) Sand - Bignoniaceae. Ciência Florestal, Santa Maria, RS, v. 15, n. 1, p. 87-92, 2005.

SILVA JUNIOR, M. C. 100 árvores do Cerrado. Brasília, DF: Rede de Sementes do Cerrado, 2005. 278 p.

STOCKMAN, A. L.; BRANCALION, P. H. S.; NOVEMBRE, A. D. L. C.; CHAMMA, H. M. C. P. Sementes de ipê-branco (Tabebuia roseo-alba (Ridl.) Sand. - Bignoniaceae): temperatura e substrato para o teste de germinação. Revista Brasileira de Sementes, Londrina, v. 29, n. 3, p. 139-143, 2007. DOI: http://dx.doi. org/10.1590/S0101-31222007000300016

TICHAVSKY, M. C. M. R. Manual de agrohomeopatía. México: Desarollo Social, 2007. 77 p. 